\title{
A parametric approach to acoustic entropy estimation for assessment of fatigue damage
}

\author{
Ali Kahirdeh ${ }^{1}$, Christine Sauerbrunn ${ }^{2}$, Huisung Yun, Mohammad Modarres \\ Center for Risk and Reliability, Department of Mechanical Engineering, University of Maryland, \\ College Park, MD, USA \\ Email: Kahirdeh@umd.edu, csauerbrunn@gmail.com, hsyun@umd.edu, Modarres@umd.edu
}

\section{Keywords:}

Acoustic Entropy, Parametric Estimation, Information Entropy, Acoustic emission, Aluminum alloys

\begin{abstract}
A parametric approach to estimating the acoustic entropy detected over the course of fatigue damage is presented. Information entropy and relative entropy is estimated through a parametric approach where trial probability density functions (PDFs) are fitted to each individual received acoustic signal as the material degrades over the cycles of loading. The PDF that produces the maximum cumulative entropy is selected to model the signals. This selection criterion is due to the fact that the PDF with higher cumulative entropy results in less bias during the selection process. The evolution trends of both information entropy and relative entropy show the stages of fatigue damage observed in the fatigue indicators such as change in hardness. The acoustic entropy has an advantage over the conventional indices of damage as it can be employed directly in the online sensor based structural health monitoring schemes as a diagnosis feature.
\end{abstract}

\section{Introduction}

Information regarding the health of the constitutive elements of the critical structures is of paramount importance to ensure catastrophic failure is avoided. Such information is obtained by probing into the underlying physics of the problem and inspecting the structures for material degradation. Almost all solid materials are prone to fatigue damage due to any cyclic loading they experience over their life. The

1 Corresponding author, Phone: +1 (301) 405 5297, Fax:+1 (301) 3149601

${ }^{2}$ Currently with Boeing Company, St. Louise, MO. USA 
problem of the deformation and damage of solid materials can be viewed as a thermodynamic process where the materials - e.g. metals or composites - under elastic or plastic loading fluctuation are considered to be a thermodynamic system. In such a procedure, the work applied on the material is dissipated due to irreversible dissipative mechanisms. A portion of the input energy is transformed into heat and acoustic dissipated energy.

The thermodynamic entropy generation has been employed as a method of damage characterization in solid materials. Of the early works that has investigated the concept of the entropy production in the course of the deformation of the solid materials, is the work of the Nobel laureate Percy Bridgman [1] who investigated the entropy production during the plastic deformation of the solids. Later in the eighties, the concept of the entropy accumulation was employed in studies related to fatigue of the materials by Whaley and coworkers $[2,3]$ who reported that the total accumulated entropy is constant at the time of the failure of the material. Of the studies in this field, the work of the Basaran and Yan [4] provides a thermodynamic framework for the damage mechanics of the materials employed in the solder joints. Basaran and Yan [4] use Boltzman entropy as a measure of the disorder in the material. For fatigue damage, the hypothesis of the constant entropy at the time of the failure was experimentally investigated by Naderi et al. [5] who reports that within the low-cycle fatigue regime, the accumulated fatigue fracture entropy at the time of the failure is constant. Their findings are for specimens under large deformation in bending mode. The experimental approach to entropy was extended to corrosion-fatigue by Imanian and Modarres who developed a reliability model based on the accumulation of the entropy [6-8]. Also this concept is examined in high cycle fatigue of the metals [9] as well as in presence of the stress concentration [10]. More studies employed the entropic approach to damage characterization of the materials in recent years [11-16]. The entropic methods to characterize the damage in solid materials are based on the measurement of the ratio of the hysteresis energy and thermal response of the material in the course of the deformation. Measurement of the hysteresis energy for a component under loading is difficult and cannot be done other than in laboratory environment employing loading frames. 
Additionally, the thermal response of the material, necessary for the calculation of the entropy, is typically insignificant for high cycle fatigue (HCF) or very high cycle fatigue range where temperature rise is minor due to insignificant plastic deformation in the material. Therefore, alternative methods of damage characterization is needed that can be employed in environments other than laboratory. One such method is acoustic emission which is a direct result of the variations in the microstructure of the material as it degrades under the cyclic loading.

The thermodynamic entropy and energy dissipation can be both employed as a measure of damage and they have been reported successfully for damage characterization in the literature [5, 16-21]. The definition of the thermodynamic entropy is based on the ratio of the strain energy over the temperature. In low cycle fatigue the temperature rise of the material is appreciable due to existence of the high amount of the plasticity in the deformation of the solid material. The trend of the evolution of the temperature profile of the metallic specimen under fatigue loading can be observed in [22]. Due to existence of the temperature term in the denominator of the entropy formula, it can be discussed that the entropy formula can better represent the evolution trend of the damage in the material in the low cycle regime comparing to the energy dissipation only. However, in high cycle fatigue problems the temperature rise in the material is not significant and therefore the evolution trend of the energy and entropy will be almost similar.

Mechanisms of damage and degradation in materials are irreversible phenomena that are accompanied by energy dissipation [17] and thermodynamic entropy generation [23]. The associated entropy generation can be viewed as an index of the resulting damage [24].The entropy generation connects the behavior of the material at the dislocation level to that of the material in the macroscopic level [25]. This is due to the fact that the entropy generation exists across all length scales $[11,25]$. The energy dissipation in the material as a result of mechanical loading can be decomposed into three mechanisms: mechanical dissipation [26], thermal dissipation [26] and acoustic dissipation. The acoustic sources within the material can be due to various mechanisms such as elastic and plastic deformation [27], phase transitions 
[28], dislocations [29], recrystallizations [27], slip [27], or twining [30]. During the mechanical loading and subsequent material damage depending on the level of the applied stress/strain, each of these damage mechanisms may evolve and result in acoustic dissipation. Any combination of these acoustic sources can be present when a material is subjected to fatigue. The acoustic waves generated during the deformation of solid materials carry information about the underlying microstructural changes inside the material, such as dislocation dynamics, defect evolution, and changes in the density of the micro- and sub-micro cracks. While these acoustic waves are inaudible, the signals can be detected, amplified, and analyzed using piezoelectric sensors attached to the material's surface.

The authors propose that acoustic entropy can be obtained by employing the information and relative entropy of the acoustic signals measured during the course of the cyclic deformation.

The acoustic entropy can represent the disorder induced in the material as a result of damage caused by microstructure changes. In the past, the entropic measure of determining regularity, i.e. approximate entropy [31], has been applied to acoustic emission signals to detect defects in rotating machineries [32]. The approximate entropy is also utilized for crack detection [33]. Unnthorsson et.al.[34] employed the information entropy of acoustic emission signals for the purpose of condition monitoring of the composite materials under cyclic loading. Their approach was to measure the entropy of the acoustic signals using estimation of the normalized histograms of the signals. In this approach, it was assumed that the frequency distribution of the signal values is constant within the signal segment [34]. Additionally, the values of the entropy in this estimation method are dependent on the binning rule chosen to create the histograms of the acoustic signal.

This paper aims at calculating the acoustic entropy generation in the course of the cyclic loading leading to fatigue damage in metallic components. Specifically, results of acoustic entropy during fatigue loading of aluminum alloy 7075-T6, a material commonly used in airframe structures, are reported. The premise of this paper is that the acoustic entropy is a fraction of the total entropy generation of the material as it 
undergoes cyclic deformation and, by itself, can be used as an index of the trend of the fatigue damage. Our approach is parametric in the sense that various PDF models are employed to model the acoustic emission signal's PDF that can describe the random variability seen in each individual acoustic signal. We aim at reducing the bias in the entropy estimation procedure by employing maximum cumulative entropy [35] in the procedure of the entropy estimation. The information entropy of the acoustic signals is estimated first by assuming that each measured acoustic signal is independent from other signals measured prior and after. In a second method, the relative entropy of acoustic signals is estimated and the PDF of each signal is estimated considering the PDF of the previously measured signal (in the step before) as prior (pre-known) information. In both approaches, the PDF that gives the maximum calculated cumulative entropy is chosen among the set of trial PDFs.

\section{Material and experimental setup}

The experimental setup consists of three parts: the specimen geometry and design, the mechanical testing machine, and the AE signal acquisition system. A dog-bone geometry is used for the Aluminum 7075-T6 specimens following ASTM standard E466. The specimen is depicted in Fig.1 and the material's property and its chemical decomposition is presented in Table 1 and Table 2, respectively. A servo-hydraulic Material Testing System (MTS) machine retrofitted with an Instron 8800 controller is used to test the specimens until crack initiation time. Crack length is monitored with an optical microscope and crack initiation is noted at the first visible change at the notch using the microscope images. An AE system including hardware and software packages supplied by the Physical Acoustics Corporation (currently part of the MISTRAS Group, Inc.) is used to record the AE signals. The hardware components consist of an AE sensor that collects the signals, a $40 \mathrm{~dB}$ preamplifier that amplifies the electrical signals according to [36], and a data acquisition module. The AE sensor, a resonance piezoelectric transducer (Mistras' Physical Acoustic Corporation Micro 30s sensor) is attached to the specimen via gel type acoustic couplant. The AE signals were filtered at a threshold value of $47 \mathrm{~dB}$. The AE timing parameters of the Peak Definition Time (PDT), Hit Definition Time (HDT) and Hit Lock Time (HLT) were set to 300, 600, 
and $1000 \mu \mathrm{s}$, respectively. The analogue filter was set a range of $1 \mathrm{kHz}$ to $3 \mathrm{MHz}$. The experimental variables of the performed tests are also presented in Table 3.

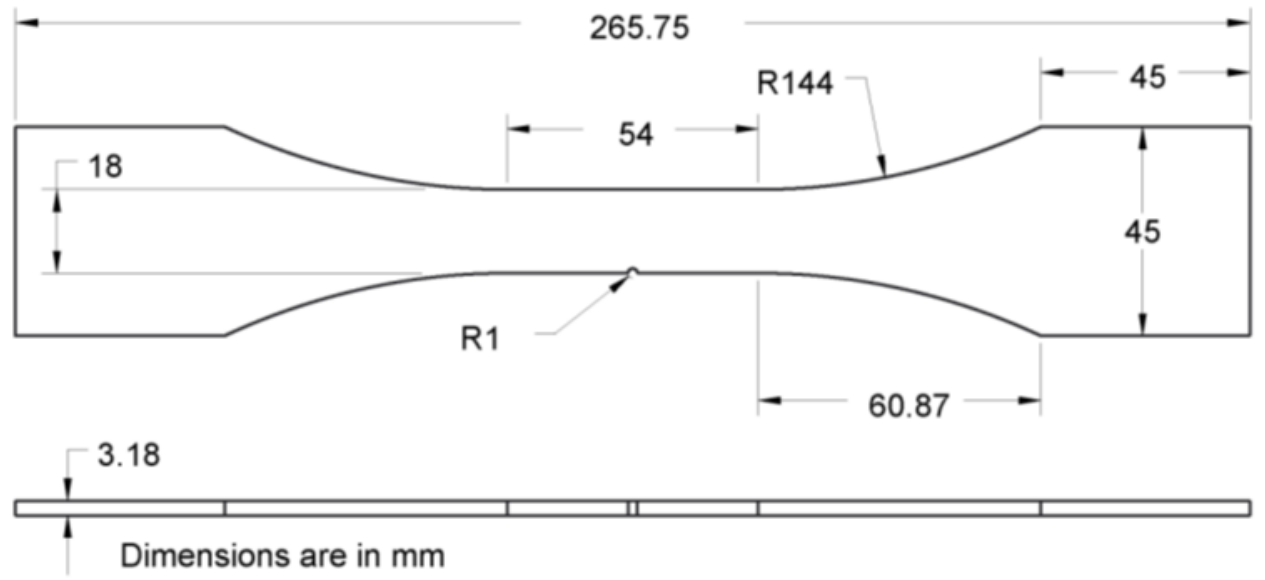

Fig. 1. The Specimen geometry

Table 1. The material properties of Al 7075-T6 used to manufacture the specimens

\begin{tabular}{lllll}
\hline $\begin{array}{l}\text { Material } \\
\text { Property } \\
\begin{array}{l}\text { Measured Value } \\
\text { from Tensile } \\
\text { Tests }\end{array}\end{array}$ & $\begin{array}{l}\text { Ultimate } \\
\text { Strength (MPa) }\end{array}$ & $\begin{array}{l}\text { Yield Strength } \\
(\mathbf{M P a})\end{array}$ & \% Elongation & $\begin{array}{l}\text { Modulus of } \\
\text { Elasticity (GPa) }\end{array}$ \\
\hline
\end{tabular}

Table 2. Chemical decomposition of the Al 7075-T6

\begin{tabular}{llllllllllllll}
\hline $\begin{array}{l}\text { Element } \\
\begin{array}{l}\text { Composition } \\
\text { (wt \%) }\end{array}\end{array}$ & $\mathbf{A l}$ & $\mathbf{Z n}$ & $\mathbf{M g}$ & $\mathbf{C u}$ & $\mathbf{C r}$ & $\mathbf{F e}$ & $\mathbf{M n}$ & $\mathbf{S i}$ & $\mathbf{T i}$ & $\mathbf{V}$ & $\mathbf{Z r}$ & Other \\
\hline
\end{tabular}

Table 3. Experimental variables of the performed tests.

\begin{tabular}{cccc}
\hline Test Number & $\begin{array}{c}\text { Applied load by the } \\
\text { loading frame (KN) }\end{array}$ & $\begin{array}{c}\text { Estimated stress at the } \\
\text { notch (MPa) }\end{array}$ & Stress ratio \\
\hline $\mathbf{1}$ & 12 & 548.5 & 0.1 \\
$\mathbf{2}$ & 14 & 639.4 & 0.1 \\
$\mathbf{3}$ & 10 & 456.7 & 0.1 \\
$\mathbf{4}$ & 13 & 593.7 & 0.1 \\
$\mathbf{5}$ & 9 & 411.0 & 0.1 \\
$\mathbf{6}$ & 12 & 548.0 & 0.1 \\
$\mathbf{7}$ & 9 & 411.0 & 0.1 \\
$\mathbf{8}$ & 10 & 456.7 & 0.1 \\
\hline
\end{tabular}




\section{Method 1: Information entropy}

The information entropy associated with the probability distribution $p(x)$ is defined as in Eq. 1 . The entropy of a random variable, $x$, describes the microscopic state of a physical system and represents the non-equilibrium entropy $[37,38]$.

$I[p(x)]=-k_{B} \int p(x) \log p(x) d x$

The measure in Eq. 1. demonstrates many properties of the entropy for the discrete random variable, introduced by Shannon [39]. However, for a continuous random variable Eq. 1 can attain infinitely large, negative and positive values [40, 41]. In a practical situation, such as the estimation of the acoustic entropy of the experimentally measured signals, estimation of the PDFs is challenging. The reason is that the governing distribution of the signals is unknown to the observer. This turns the problem of the entropy estimation to a problem of assigning a distribution to the values of the acoustic waveforms in a way that the procedure is unbiased. The intuitive way to assign a less biased probability distribution to the waveforms is based on the fact that a wide distribution is of more uncertainty and less bias than a peaked distribution. Therefore, in such inference processes where one has no information on the form of the probability distribution, we must choose the PDF that has "maximum entropy subject to whatever is known. This is the only unbiased assignment we can make" according to E.T. Jaynes [35].

The problem now is to select an appropriate PDF among the trial PDFs. This problem lies in the category of the parametric PDF estimation. In the parametric PDF estimation, a strong assumption is usually made regarding the form of the underlying scatter of the data. In this case, we aim at reducing the level of the bias in selection and limit making assumptions regarding the governing PDF of the data. For this we employ the distribution that has the maximum cumulative entropy to select the model that better represents the data. This approach can be seen as a "model selection" approach where you have a number of models in hand, however, there is no prior knowledge regarding which model can best represent the 
data. The problem to be solved is to identify the probability density function, $f(x)$, subject to the $\int_{-\infty}^{+\infty} f(x) d x=1$ that maximize the entropy as defined by Eq. 1.

In the current approach to estimate the PDF of the acoustic emissions signals, a number of representative PDFs of the waveforms as trial models are arbitrarily chosen. The maximum likelihood estimation (MLE) of parameters of these PDFs given the acoustic signals is used. The PDF model that gives the maximum cumulative entropy is chosen as the plausible model for estimating the entropy of the acoustic waveforms. The PDFs that are candidates for modeling the acoustic signals are Normal [42], Extreme value [43], Exponential [44], Logistics [45], Weibull [44], Nakagami [46], Lognormal [47] and Rayleigh [47]. Once the MLE of the parameters are obtained, Eq. 1 is numerically integrated using the global adaptive quadrature in the MATLAB environment.

\subsection{Information entropy results}

Figure 2 represents the entropy and its cumulative form for a fatigue experiment conducted under the maximum loading of $12 \mathrm{kN}$, loading ratio of 0.1 and the loading frequency of $5 \mathrm{~Hz}$.

Eight trial PDFs are employed to estimate the information entropy of the acoustic emission signals. In estimating the entropy of the signals, each signal is assumed to be an independent event. A PDF is fitted to the voltage values of the acoustic emission waveforms, and the parameters of the PDF are estimated using the MLE method. Once the parameters of the PDF are known, the entropy is estimated using Eq. 1. The coefficient $\mathrm{k}_{\mathrm{B}}$ is neglected from the calculation in this case for following the notation of the information entropy. In Fig. 2, each individual blue dot represents the entropy of a signal and the green curve represents the cumulative form of entropy. The trend of evolution of the cumulative entropy obtained from different probability density functions demonstrates similar trend of evolution. However, the order of the magnitudes of the estimated entropies is different due to differences in the mathematical definition of the various PDFs. The absolute value of the cumulative acoustic entropy is shown in Fig. 3 for eight different PDFs. It is observed that the exponential distribution exhibits the largest amount of 
entropy. In Fig. 3a, the trend of the entropy evolution for the PDFs that gives smaller entropy is affected by those have large cumulative entropy and as a results trend of evolution for the Weibull distribution and Normal distribution cannot be identified in the Fig.3a. In order to compare the trend of evolution of the entropy for each PDF, the standardized plot of each cumulative entropy is depicted in Fig. 3b. The standardized form of the data, cumulative entropy, is defined by the standard normal random variable $\mathrm{z}=\frac{\mathrm{x}-\mu}{\sigma}$, where $\mathrm{x}$ is the random variable with $\mu$ as representing mean of the data, and $\sigma$ is the standard deviation of the data. We look into the standardized representation of the data where the mean of the cumulative acoustic entropy is removed from each data set and is divided by the standard deviation, depicted in Fig. 3b. The trend observed in the standard cumulative entropy depicts the trend of the fatigue damage evolution. In order to estimate the information entropy of the acoustic emission signals parametrically, the procedure is to first estimate the parameters of an exponential distribution using the MLE and then estimate the information entropy accordingly. The entropy formula for an acoustic signal modeled with an exponential distribution is given by Eq. 2. It should be noted that the absolute positive values of the signals are considered for the estimation of the PDF of the acoustic signal.

$S=-\int_{0}^{+\infty}\left(\frac{1}{\mu} e^{-\frac{x}{\mu}}\right) \log \left(\frac{1}{\mu} e^{-\frac{x}{\mu}}\right) d x$

where $\mu$ is the parameter of the exponential distribution. 

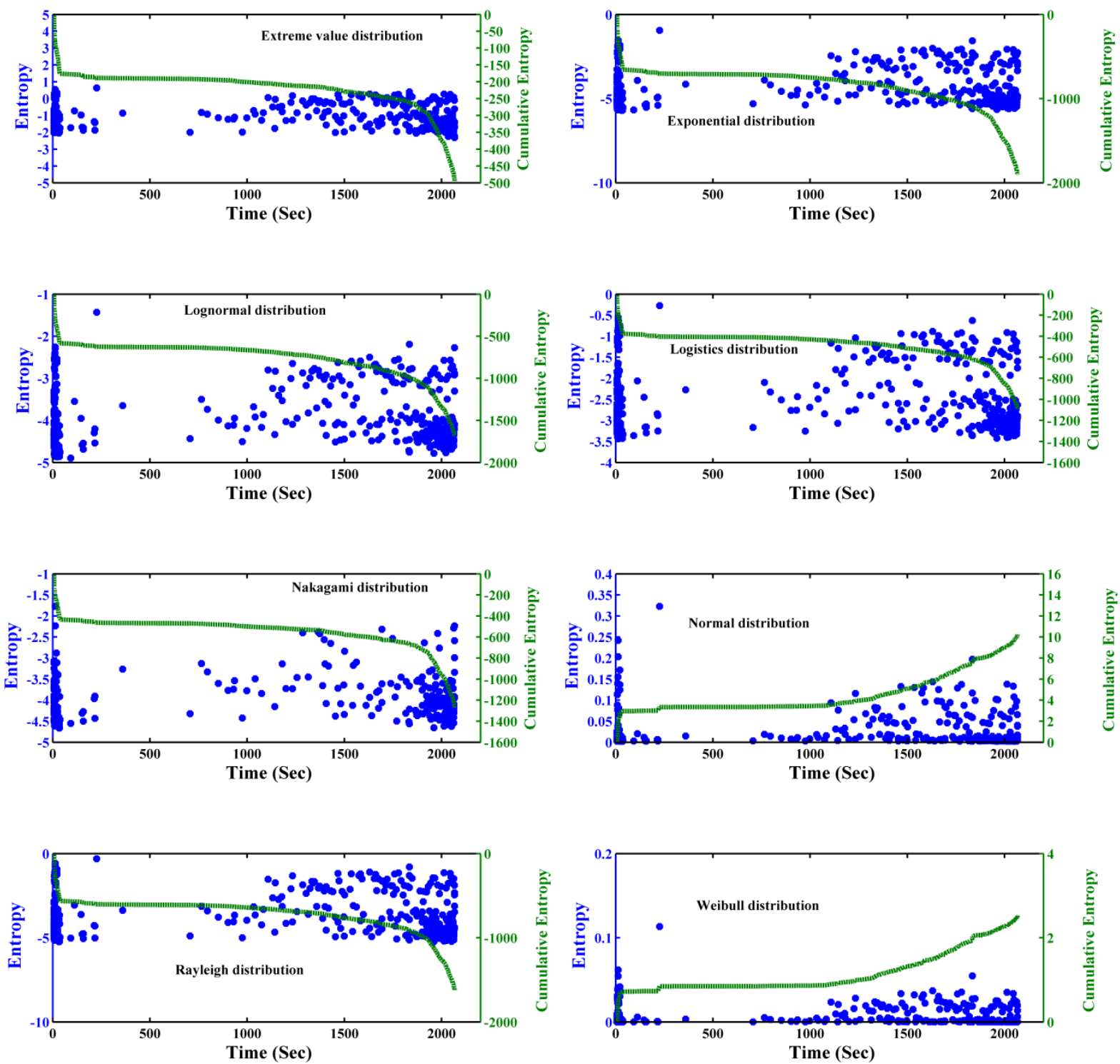

Fig.2. Parametrically estimated information entropy and its cumulative form for eight trial PDFs. The procedure is that eight PDFs are candidates for modeling the underlying distribution of the acoustic emission waveforms. 

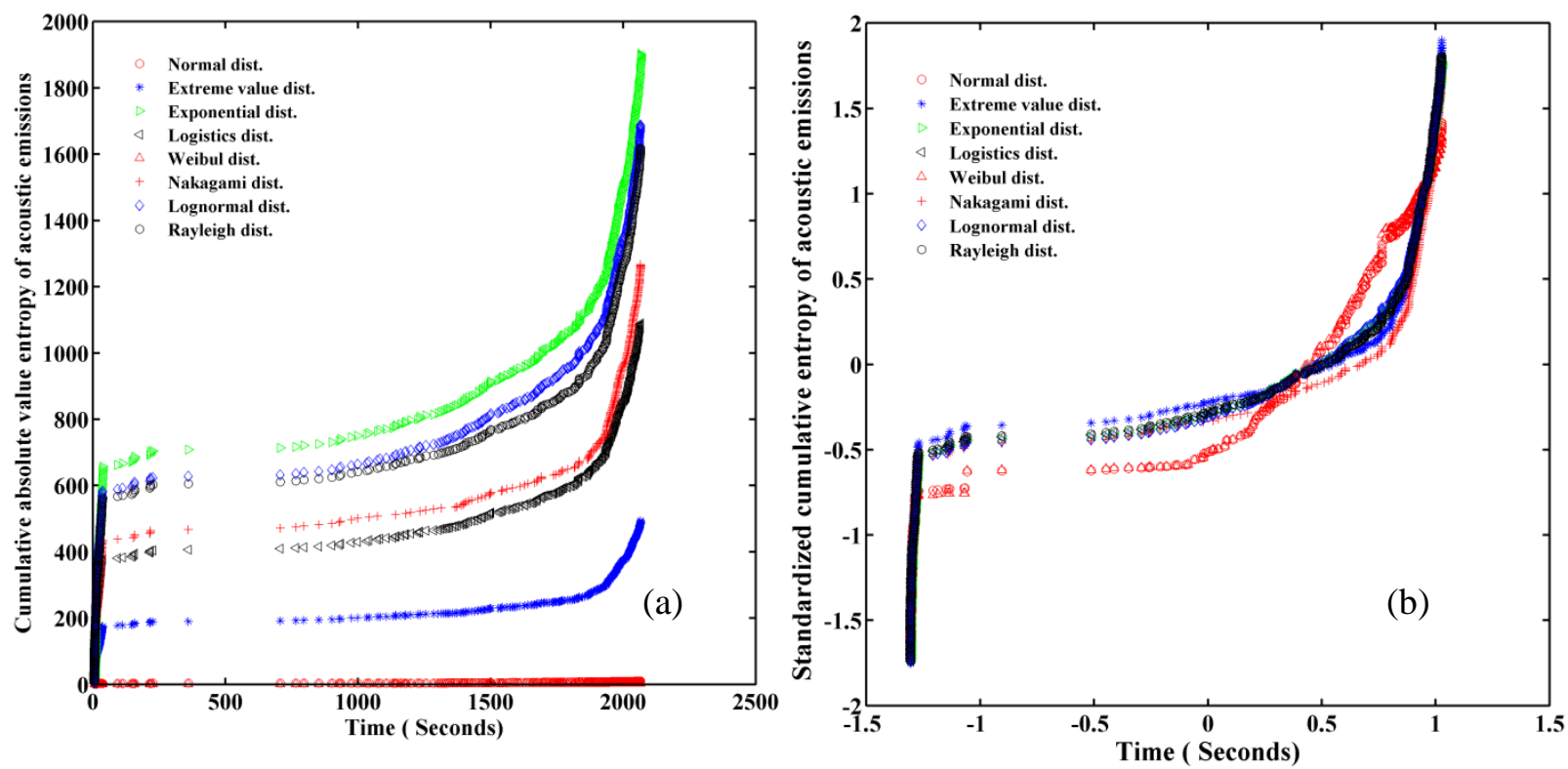

Fig. 3a The absolute value of the estimated acoustic emission information entropy. As it is apparent the maximum value of the entropy is obtained by the selecting the underlying model of the signals as exponential distribution. In Fig. 3a, the graphs related to Weibull distribution and Normal distribution are plotted on each other close to $\mathrm{x}$ axis and therefore are not distinguishable. Fig. 3b, The standardized cumulative acoustic emission entropy where the eight graphs related to eight trial probability density function are plotted.

\section{Method 2: Relative entropy}

In the previous section, it was assumed in the analysis that each individual measured acoustic signal is an independent event from other acoustic events. The information derived from an acoustic signal, and the probability distribution that is fitted to that measured signal can serve as prior information for estimation of the entropy of the next measured signal. Now, we employ relative entropy of the two consecutive PDFs to estimate the entropy of the acoustic signals with the assumption that each acoustic event provides prior information regarding the next acoustic event.

Given two probability distributions of $p$ and $q$ on the space of the states $X$, the relative entropy is defined as in Eq. 3 [48].

$S(p \mid q)=-\sum_{x \in X} p(x) \log \left(\frac{p(x)}{q(x)}\right)$ 
$S(p \mid q)$ is always negative and attains a zero value if $p=q[49]$. For probability density functions of continuous variables, the relative entropy is defined as in Eq. 4.

$S(P \mid Q)=-\int_{-\infty}^{+\infty} p(x) \log \left(\frac{p(x)}{q(x)}\right) d x$

where $p(x)$ and $q(x)$ denotes the PDFs of the random variable $x^{3}$. In our parametric approach to estimating the acoustic entropy, the relative entropy of the two consecutive distributions are estimated using Eq. 4. We specify the index, $i$, to the occurrence of each individual acoustic event and define the relative entropy between two consecutive events as in Eq. 5 .

$S_{i}(P \mid Q)=-\int_{-\infty}^{+\infty} p_{i}(x) \log \left(\frac{p_{i}(x)}{p_{i-1}(x)}\right) d x$

where the $i$ corresponds to the current received signal and $i-1$ corresponds to the previously received signal. In this fashion, we use the information of the PDF of the acoustic signal of each step of the experiment in the estimation of the entropy of the current step of the experiment. In the physical systems of an aluminum alloy sample under the cyclic fatigue loading, the source of information about the microstates of the material are the acoustic events. As we receive new signals that describes the acoustic dissipation state of the material, we update the statistical states of the system described by $p(x)$ to $p(x \mid y)$ where $y$ translates the new information obtained on the microstates of the material carried by the acoustic signals.

\subsection{Results of relative entropy}

Figure 4 demonstrates the parametric estimation of the relative entropy of the acoustic signals and its cumulative form. Eight different probability density functions are employed to estimate the acoustic emission relative entropy. All of the estimated relative entropies attain negative values due to the negative sign behind the integral in the Eq. 4 and 5 and the fact the Kullback-Leibler measure of distance between

3 The relative entropy is also known as Kullback-Leibler measure of distance between the two states of random variable $\mathrm{x}$ represented by two PDFs $\mathrm{q}(\mathrm{x})$ and $\mathrm{p}(\mathrm{x})$. The definition of the Kullback-Leibler is without the minus sign behind the integral which result into a non-negative measure of the distance between the two distributions. 
two PDFs is always positive [50]. Figure 5a demonstrates the absolute value of the estimated cumulative relative entropy and it can be observed that Rayleigh distribution [51] gives the maximum cumulative entropy of the acoustic emission signals over the course of the cyclic fatigue deformation. In Fig. $5 \mathrm{~b}$ the standardized version of Fig. 5a is depicted to reveal the underlying trend of evolution of the acoustic emission cumulative relative entropy. The trend of evolution of cumulative acoustic emission relative entropy demonstrates the trend of evolution of the damage in the tested materials. The Rayleigh distribution is selected to model the acoustic emission signals and estimate the probability density function based on which the relative entropy is calculated. The relative entropy formula for an acoustic signal whose probability density function is modeled by a Rayleigh distribution [51] is obtained as in Eq.

6.

$S=-\int_{0}^{+\infty}\left(\frac{x}{b_{x}^{2}} e^{\left(-\frac{x^{2}}{2 b_{x}^{2}}\right)}\right) \log \left(\frac{b_{y}^{2} e^{\left(-\frac{x^{2}}{2 b_{x}^{2}}\right)}}{b_{x}^{2} e^{\left(-\frac{x^{2}}{2 b_{y}^{2}}\right)}}\right) d x$

where $b_{x}$ is the parameter of the Rayleigh distribution at the current instant of time (the current received acoustic signal) and the $b_{y}$ is the parameter of the of the Rayleigh distribution of the previously measured acoustic signal (prior information). 

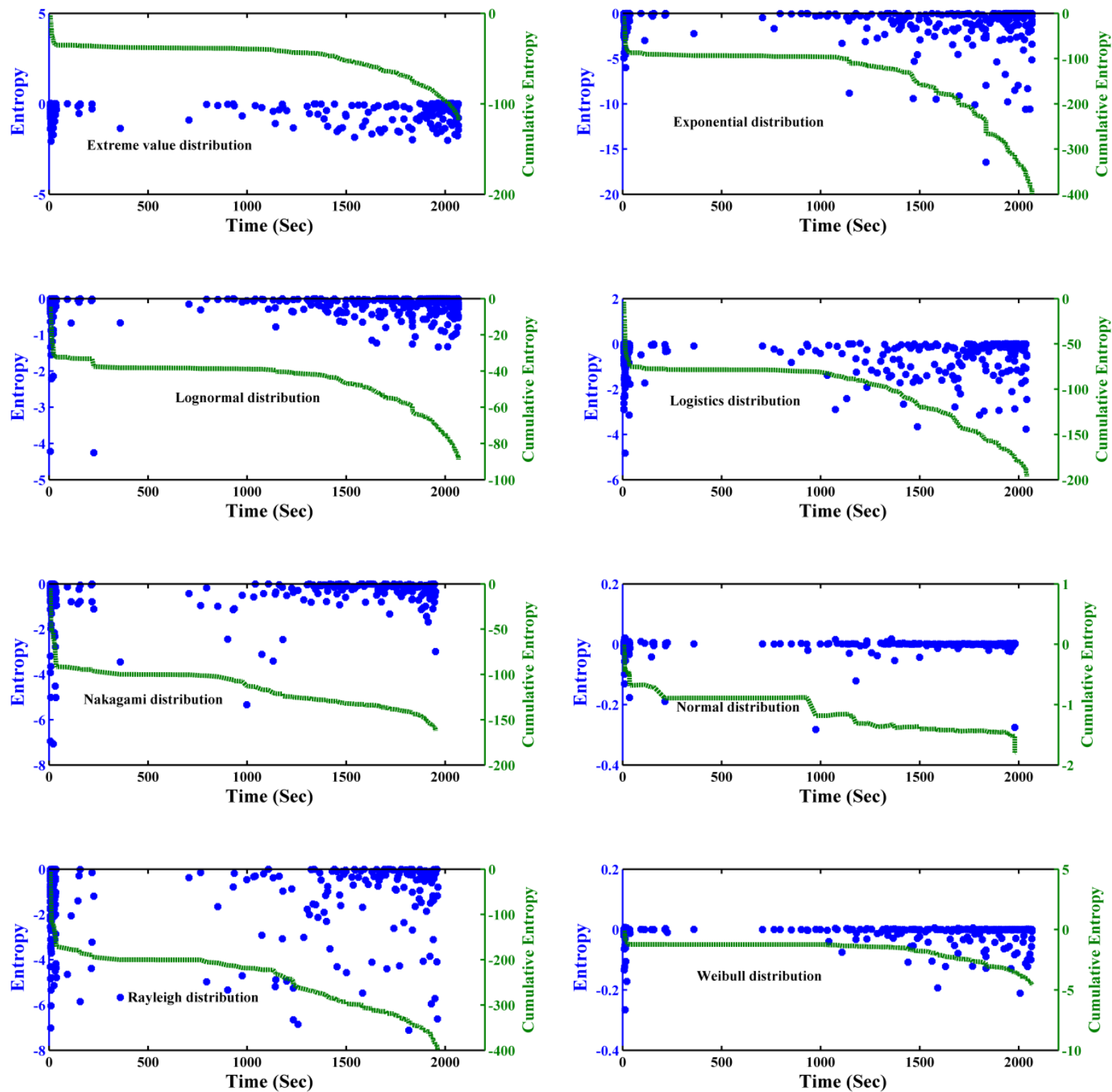

Fig. 4. Parametrically estimated relative entropy and its cumulative form for eight trial PDFs. The procedure is that eight PDFs are candidates for modeling the underlying distribution model of the acoustic emission waveforms. The relative entropy of each signal is estimated having the fitted distribution of the previously measured acoustic emission event. Therefore, the estimated entropy at each instant of time, the time at which an acoustic signal is received, is dependent on the previously measured signal. 

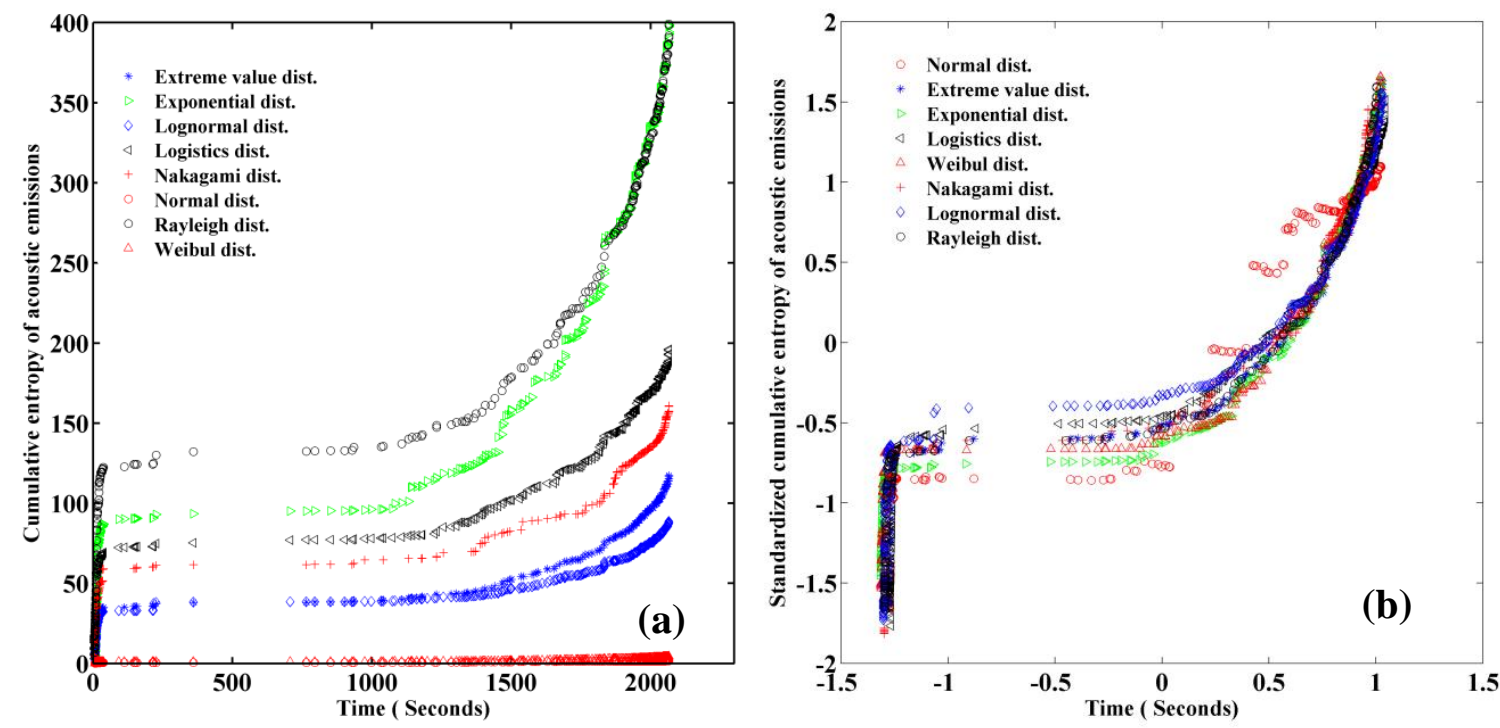

Fig. 5a The absolute value of the cumulative relative entropy for the eight trail probability density functions. It is observed that the Rayleigh distribution gives the maximum estimate for the relative entropy of the acoustic emissions signals and consequently provides the least bias in the selection of a model for the probability distribution. In Fig. 5a, the graphs related to Weibull distribution and Normal distribution are plotted on each other close to $\mathrm{x}$ axis and therefore are not distinguishable. Fig. 5b, The standardized plot of the cumulative relative entropy.

\section{Comparison to hardness change}

It is aimed to compare the trend of evolution of the cumulative acoustic entropy with other metrics of the degradation. The metric that is employed in the literature as a measure of crack initiation is change in the hardness [52]. We compare our results of the cumulative acoustic entropy with the changes in the hardness during the crack initiation phase. We employ the results reported by Pavlou [52] to compare with cumulative acoustic entropy. In the study performed by Pavlou [52] a phenomenological nonlinear fatigue damage accumulation rule is introduced based on the changes in the hardness of the Al 2024-T42. In this study the experimental normalized Vickers hardness change is reported for different stress ranges of 125-225 MPa. Although this material is not similar to what we have tested, Al 7075-T6, it is expected that the evolution trend of the damage accumulation for these two aerospace grade alloys are close enough to compare. Figure 6 demonstrate the change in the hardness as a function of the consumed life of the specimens under the fatigue loading. It is apparent that the tests in higher stress amplitude are of shorter life. The nonlinear trend of evolution of damage is obvious in the results of the hardness change. 
Since the tests in Fig. 6a are of different consumed life, the trend of evolution of the tests of higher amount of stress is not clearly noticeable. Therefore, in order to reveal the trend better, we remove the mean of the hardness change in each experiment and divide that by the standard deviation of the corresponding experiment to build the standardized hardness change. This representation of the data helps to have a better visual comparison of the evolution trends. Such trends are demonstrated in Fig. $6 \mathrm{~b}$. Comparing the trends observed in the Figs. $3 \mathrm{~b}, 5 \mathrm{~b}$, and $6 \mathrm{~b}$, it can be concluded the both methodologies reveal similar evolution trend.
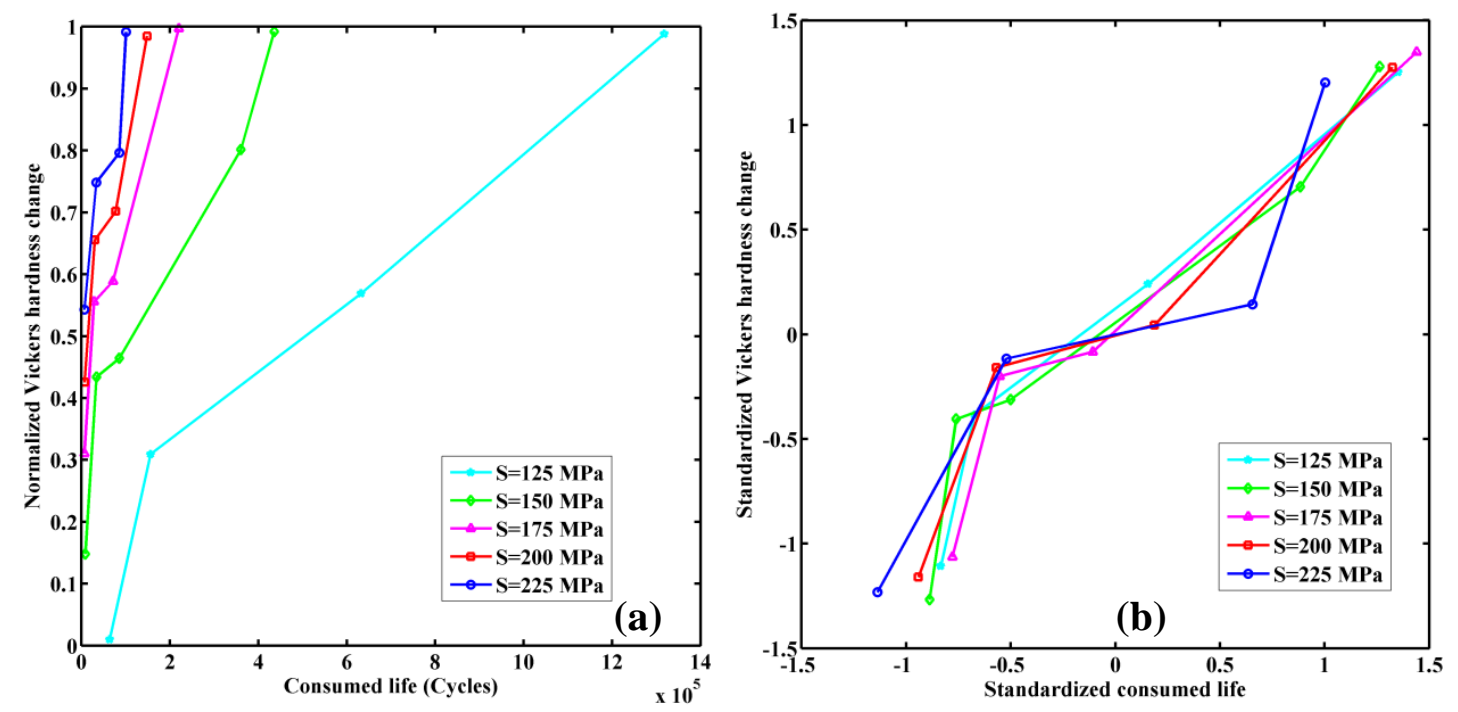

Fig. 6a, Hardness change in Al 2024-T42, Fig. 6b, Standardized hardness change in Al 2024-T42. The data of the hardness change are obtained from the study by Pavlou [52].

\section{Conclusions and discussions}

In this research, a parametric approach to estimating the acoustic entropy is presented. Acoustic entropy is introduced as a fraction of the total entropy production of the materials in the course of the deformation cycles. As materials accumulate damage under fluctuating loading, they dissipate the input work through several dissipation mechanisms including mechanical hysteresis, thermal dissipation, and acoustic dissipation. The information entropy of the acoustic emissions is estimated parametrically employing two different approaches. The first approach assumes that the received acoustic events are independently and 
identically distributed and the acoustic entropy is estimated using information entropy of the acoustic signals. In this approach, the parameters of eight trial PDF models are estimated and the PDF that gives the maximum cumulative information entropy is chosen to calculate the probability distribution needed for estimation of the information entropy. Results show that the exponential distribution provides the maximum cumulative information entropy among the trial set of PDFs for all of the measured acoustic signals in the course of the fatigue degradation.

In the second approach, the acoustic events measured during the fatigue experiment are considered as dependent events in the sense that each individual measured acoustic signal is related to the previously measured acoustic signal. In this approach the relative entropy is employed for estimation of the acoustic entropy during the fatigue damage of the material. The procedure is similar to the previous approach. Eight trial PDFs are employed to model each individual measured acoustic emission event. This gives the probability distribution needed for estimation of the entropy. Once the probability distribution is obtained, the relative entropy is employed for estimation of the acoustic entropy. The relative entropy makes use of the PDF of the previously measured acoustic signal and the current measured signal to obtain the entropy. Results show that the Rayleigh distribution gives the maximum cumulative relative entropy among the trial set of PDFs for the acoustic signals measured in the course of the fatigue.

Since the microstructure of the material at each instant of the deformation or time instant under the cyclic loading is dependent on the previous state of the deformation, therefore such assumption can also hold for the acoustic emission signals which are generated as a result of the microstructure deformation. Therefore, the relative entropy provides a more realistic assumption.

In this paper, fatigue degradation is viewed in the form of disorder in materials. Such disorder is in terms of changes in microstructural properties and manifests itself in form of the acoustic emissions signals that can be quantified using information entropy. In this perspective, the probability density function of voltage signals as opposed to the conventional features of acoustic emissions that depend on the voltage 
values of the signals is used. In employing acoustic emissions for health monitoring of materials and structures, many factors such as operational parameters, environmental conditions as well as material properties affect the acoustic emission sensor's output. As such, features that provide information on the deterioration of the probability distributions of the signals can result in information regarding the underlying damage of the material. It is worth noting that the mean stress can have an effect on the acoustic emissions. In this study we have not tested the methodology under several mean stresses. It is expected that the cumulative acoustic entropy exhibit similar trend of evolution under different mean stress. While other forms of the energy dissipation and entropy generation may not be practically measureable in the course of the cyclic deformation of the structures in environment other than laboratory, the acoustic entropy can be measured using acoustic emission sensors.

\section{Acknowledgements}

This work is part of ongoing research through grant number N00014140453 from the Office of Naval Research (ONR). The authors would like to acknowledge the constructive comments from two anonymous reviewers of the earlier version of this paper that improved the paper substantially.

\section{References}

[1] P. Bridgman, The thermodynamics of plastic deformation and generalized entropy, Reviews of Modern physics, 22 (1950) 56.

[2] P.W. Whaley, A Mathematical Model for Internal Friction and Local Fatigue Damage Based on Populations of Yielding Microelements, Journal of Vibration, Acoustics, Stress, and Reliability in Design, 109 (1987) 201-206.

[3] P.W. Whaley, P.S. Chen, G.M. Smith, Continuous measurement of material damping during fatigue tests, Exp Mech, 24 (1984) 342-348.

[4] C. Basaran, C.Y. Yan, A Thermodynamic Framework for Damage Mechanics of Solder Joints, Journal of Electronic Packaging, 120 (1998) 379-384.

[5] M. Naderi, M. Amiri, M.M. Khonsari, On the thermodynamic entropy of fatigue fracture, Proceedings of the Royal Society of London A: Mathematical, Physical and Engineering Sciences, 466 (2010) 423438.

[6] A. Imanian, M. Modarres, An Entropy-Based Theory of Reliability with the Application to Corrosion Fatigue,, in: European Safety and Reliability Conference (ESREL2015) Zürich, Switzerland, , 2015. 
[7] M. Modarres, A. Imanian, Development of a Generalized Entropic Framework for Damage Assessment with Application to Corrosion Fatigue, in: ASME 2015 Applied Mechanics and Materials Conference, McMAT2015, Seattle WA., 2015.

[8] A. Imanian, M. Modarres, Thermodynamics as a fundamental science of reliability, Proceedings of the Institution of Mechanical Engineers, Part O: Journal of Risk and Reliability, 230 (2016) 598-608.

[9] M. Liakat, M.M. Khonsari, On the anelasticity and fatigue fracture entropy in high-cycle metal fatigue, Materials \& Design, 82 (2015) 18-27.

[10] M. Liakat, M.M. Khonsari, Entropic characterization of metal fatigue with stress concentration, International Journal of Fatigue, 70 (2015) 223-234.

[11] T. Temfack, C. Basaran, Experimental verification of thermodynamic fatigue life prediction model using entropy as damage metric, Materials Science and Technology, 31 (2015) 1627-1632.

[12] A. Cuadras, J. Crisóstomo, V.J. Ovejas, M. Quilez, Irreversible entropy model for damage diagnosis in resistors, Journal of Applied Physics, 118 (2015) 165103.

[13] A. Cuadras, F. Meinert, B. P, schel, O. Kanoun, Online cellphone battery entropy measurement for SoH estimation, in: 2016 13th International Multi-Conference on Systems, Signals \& Devices (SSD), 2016, pp. 258-261.

[14] Y.-J. Sun, L.-S. Hu, Assessment of Low Cycle Fatigue Life of Steam Turbine Rotor Based on a Thermodynamic Approach, Journal of Engineering for Gas Turbines and Power, 134 (2012) 064504064504.

[15] K. Pervaiz Fathima, J. Chandra Kishen, Prediction of Fatigue Life in Plain Concrete Using Entropy Production, Journal of Engineering Mechanics, 141 (2015) 04015007.

[16] V. Ontiveros, M. Amiri, A. Kahirdeh, M. Modarres, Thermodynamic entropy generation in the course of the fatigue crack initiation, Fatigue \& Fracture of Engineering Materials \& Structures, (2016) n/a-n/a.

[17] A. Kahirdeh, M.M. Khonsari, Energy dissipation in the course of the fatigue degradation:

Mathematical derivation and experimental quantification, International Journal of Solids and Structures, 77 (2015) 74-85.

[18] M. Naderi, A. Kahirdeh, M.M. Khonsari, Dissipated thermal energy and damage evolution of Glass/Epoxy using infrared thermography and acoustic emission, Composites Part B: Engineering, 43 (2012) 1613-1620.

[19] D.S. Tchankov, K.V. Vesselinov, Fatigue life prediction under random loading using total hysteresis energy, International Journal of Pressure Vessels and Piping, 75 (1998) 955-960.

[20] M. Naderi, M.M. Khonsari, On the role of damage energy in the fatigue degradation characterization of a composite laminate, Composites Part B: Engineering, 45 (2013) 528-537.

[21] L.A. Sosnovskiy, S.S. Sherbakov, Mechanothermodynamic Entropy and Analysis of Damage State of Complex Systems, Entropy, 18 (2016) 268. 
[22] M. Naderi, M.M. Khonsari, A thermodynamic approach to fatigue damage accumulation under variable loading, Materials Science and Engineering: A, 527 (2010) 6133-6139.

[23] M.D. Bryant, M.M. Khonsari, F.F. Ling, On the thermodynamics of degradation, Proceedings of the Royal Society of London A: Mathematical, Physical and Engineering Sciences, 464 (2008) 2001-2014.

[24] A. Imanian, M. Modarres, A Thermodynamic Entropy Approach to Reliability Assessment with Applications to Corrosion Fatigue, Entropy, 17 (2015) 6995-7020.

[25] C. Basaran, S. Nie, J. Gomez, E. Gunel, S. Li, M. Lin, H. Tang, C. Yan, W. Yao, H. Ye, Thermodynamics Theory for Damage Evolution in Solids, in: G.Z. Voyiadjis (Ed.) Handbook of Damage Mechanics, Springer New York, 2015, pp. 721-762.

[26] J. Lemaitre, J.L. Chaboche, Mechanics of Solid Materials, Cambridge University Press, 1994.

[27] D. Drozdenko, J. Bohlen, F. Chmelík, P. Lukáč, P. Dobroň, Acoustic emission study on the activity of slip and twin mechanisms during compression testing of magnesium single crystals, Materials Science and Engineering: A, 650 (2016) 20-27.

[28] B. Jordi, M.-O. José-María, R. Francisco Javier, G. María Carmen, K.H.S. Ekhard, V. Eduard, P. Antoni, Avalanche correlations in the martensitic transition of a $\mathrm{Cu}-\mathrm{Zn}-\mathrm{Al}$ shape memory alloy: analysis of acoustic emission and calorimetry, Journal of Physics: Condensed Matter, 26 (2014) 125401.

[29] M.A. Lebyodkin, I.V. Shashkov, T.A. Lebedkina, K. Mathis, P. Dobron, F. Chmelik, Role of superposition of dislocation avalanches in the statistics of acoustic emission during plastic deformation, Physical Review E, 88 (2013) 042402.

[30] A. Vinogradov, A. Lazarev, M. Linderov, A. Weidner, H. Biermann, Kinetics of deformation processes in high-alloyed cast transformation-induced plasticity/twinning-induced plasticity steels determined by acoustic emission and scanning electron microscopy: Influence of austenite stability on deformation mechanisms, Acta Materialia, 61 (2013) 2434-2449.

[31] S.M. Pincus, Approximate entropy as a measure of system complexity, Proceedings of the National Academy of Sciences, 88 (1991) 2297-2301.

[32] Y. He, X. Zhang, Approximate Entropy Analysis of the Acoustic Emission From Defects in Rolling Element Bearings, Journal of Vibration and Acoustics, 134 (2012) 061012-061012.

[33] L. Lin, F. Chu, Approximate entropy as acoustic emission feature parametric data for crack detection, Nondestructive Testing and Evaluation, 26 (2011) 119-128.

[34] R. Unnthorsson, T.P. Runarsson, M.T. Jonsson, AE entropy for the condition monitoring of CFRP subjected to cyclic fatigue, Journal of Acoustic Emission, 26 (2008) 262-270.

[35] E.T. Jaynes, Information Theory and Statistical Mechanics, Physical Review, 106 (1957) 620-630.

[36] T.M. Roberts, M. Talebzadeh, Acoustic emission monitoring of fatigue crack propagation, Journal of Constructional Steel Research, 59 (2003) 695-712.

[37] C. Jarzynski, Equalities and Inequalities: Irreversibility and the Second Law of Thermodynamics at the Nanoscale, Annual Review of Condensed Matter Physics, 2 (2011) 329-351. 
[38] J.M. Parrondo, J.M. Horowitz, T. Sagawa, Thermodynamics of information, Nature Physics, 11 (2015) 131-139.

[39] C.E. Shannon, A mathematical theory of communication, Bell System Technical Journal, The, 27 (1948) 379-423.

[40] K. Conrad, Probability distributions and maximum entropy, retrieved November, 14 (2013) 2013.

[41] M.T. Martín, A. Plastino, V. Vampa, A Maximum Entropy Approach for Predicting Epileptic TonicClonic Seizure, Entropy, 16 (2014) 4603-4611.

[42] R.A. Johnson, D.W. Wichern, Applied multivariate statistical analysis, Prentice hall Englewood Cliffs, NJ, 1992.

[43] E.J. Gumbel, Statistics of extremes, Courier Corporation, 2012.

[44] N.L. Johnson, A.W. Kemp, S. Kotz, Univariate discrete distributions, John Wiley \& Sons, 2005.

[45] N. Balakrishnan, Handbook of the logistic distribution, CRC Press, 2013.

[46] W.C. Hoffman, Statistical methods in radio-wave propagation, (1958).

[47] C. Forbes, M. Evans, N. Hastings, B. Peacock, Statistical distributions, John Wiley \& Sons, 2011.

[48] R.C. Tolman, The principles of statistical mechanics, Courier Corporation, 1938.

[49] B. Gaveau, L. Schulman, A general framework for non-equilibrium phenomena: The master equation and its formal consequences, Physics Letters A, 229 (1997) 347-353.

[50] S. Kullback, R.A. Leibler, On Information and Sufficiency, The Annals of Mathematical Statistics, 22 (1951) 79-86.

[51] A. Papoulis, S.U. Pillai, Probability, random variables, and stochastic processes, McGraw-Hill, 2002.

[52] D.G. Pavlou, A phenomenological fatigue damage accumulation rule based on hardness increasing, for the 2024-T42 aluminum, Engineering Structures, 24 (2002) 1363-1368. 